\title{
Aprender a trabajar con las familias en Atención Temprana: estudio de caso
}

\section{Learning to work with Families with Early Intervention Support: A case study}

\author{
$\mathrm{M}^{\mathrm{a}}$ José Mayorga-Fernández ${ }^{1}$, Dolores Madrid-Vivar ${ }^{1} \mathrm{y}$ \\ $\mathrm{M}^{\mathrm{a}}$ Pilar García-Martínez ${ }^{2}$ \\ ${ }^{1}$ Universidad de Málaga, España \\ ${ }^{2}$ CAIT AMAPPACE Málaga, España
}

Disponible online 31 de agosto de 2015

\begin{abstract}
El objetivo de este estudio es conocer las necesidades de las familias que acuden a un Centro de Atención e Intervención Temprana generalista, ya que los profesionales han detectado que la oferta para su atención tiene escasa participación. Para identificar estas necesidades se ha elaborado un instrumento de recogida de información. Han participado 58 familias. Se ha realizado un análisis descriptivo de los datos, para posteriormente realizar un análisis inferencial. Estas familias en un $55.70 \%$ afirman que la propia entidad de atención temprana tendría que facilitar espacios para ese intercambio, es más, para un $67.24 \%$ dichos espacios tendrían que ser permanentes. Poner en marcha un programa de apoyo para y entre familias puede ser más fácil desde un centro de atención e intervención temprana, ya que las familias en este momento están totalmente volcadas en sus hijos/as, y es a través de la atención a sus hijos/as como se puede facilitar a las familias los apoyos necesarios, brindándoles espacio emocional y de comunicación entre familias con más experiencia, y nuevas familias que están iniciando este duro recorrido. El desafío ahora es diseñar e implementar este programa.
\end{abstract}

Palabras Clave: Centro de Atención e Intervención Temprana; Apoyo a las Familias; Detección de Necesidades.

The main aim of the present case study was to identify the needs of families attending Childhood Early Intervention Centres, because professionals working in this setting have noted the low level of participation in the programs offered. An investigation tool was designed to gather information on their needs. In total, 58 families participated in the project. A first descriptive analysis of the data was conducted, followed by an inferential one. Of these families, $55.7 \%$ considered the Childhood Early Intervention Centre to be the right setting to foster participation, and $67.24 \%$ considered that these spaces should be permanent. Implementing a support program for families and between families would be easier in a Childhood Early Intervention Centre, because parents focus on their children and their needs when they attend these centres. These support programs can be used to provide the needed support and the emotional and communication space in order to network "expert" families and families facing such issues for the first time. An upcoming challenge is to design and implement such a support program.

Key Words: Childhood Early Intervention Centers; Family Support; Needs Detection.

Correspondencia: Dolores Madrid-Vivar. Facultad de Ciencias de la Educación. Universidad de Málaga, Campus de Teatinos s/n, 29071, Málaga. E-mail: 1madrid@uma.es. E-mail de las co-autoras: Mª José Mayorga-Fernández, mjmayorga@uma.es; Ma Pilar García-Martínez: p7gama@hotmail. com. 
La atención temprana ha ido evolucionando hacia un concepto integrador de las actuaciones sociales, médicas y educativas, dirigidas a garantizar la igualdad de oportunidades de niños/as con necesidades. Es una actividad muy compleja en la que es totalmente necesario un trabajo colaborativo de las distintas instancias fundamentales en el desarrollo del niño/a, siendo una de ellas la familia (Aranda y De Andrés, 2004). La familia es una institución especializada en garantizar necesidades psicológicas, de niños/as y adultos, y de gran importancia para la socialización de los menores (Trenado, Pons-Salvador y Cerezo, 2009) cuando las pautas de crianza son adecuadas. Por ello, la familia constituye el núcleo central y fundamental al que llega un niño/a recién nacido, ya que hay que entender el desarrollo humano como una actividad social en la que los niños/as participan desde que nacen de forma activa de acuerdo con sus competencias y con la ayuda de los adultos o de compañeros más experimentados (Giné et al., 2009). Pero esta llegada, en ocasiones suele ser traumática, en el caso de que ese menor presente dificultades en su desarrollo. Para las familias, normalmente, resulta un duro golpe aceptar que poseen un niño/a con algún tipo de dificultad, dan lugar a una serie de actitudes como rechazo, negación, aislamiento, búsqueda (Cunninghan, 1995). La noticia necesita de todo un proceso de asimilación y acomodación para poder hacer frente a la nueva situación, aunque no hay que olvidar que las familias son unidades dinámicas que evolucionan (Silvestre, 2010). Cada familia suele afrontar la noticia de una forma determinada, siendo un núcleo natural al que llega un niño/a tras su nacimiento.

En los casos en que el recién llegado presenta una dificultad la familia "se rompe por dentro, aparecen una serie de emociones que les bloquean y se despiertan sentimientos de incapacidad para la crianza de ese niño/a, pero a la vez se desarrolla el firme compromiso de hacer todo lo que esté en sus manos para sacarlo adelante" (Díez, 2008, p. 46). Por ello se puede afirmar, que intervenir en atención temprana no es solo actuar directamente con los menores sino con todos aquellos ambientes y contextos que ejercen influencia directa en los mismos. Trabajar con las familias desde una perspectiva orientada al progreso y a la calidad de vida potencia en gran medida el desarrollo de los niños/as con necesidades (Giné et al., 2009).

Dada la crucial importancia de la calidad de vida familiar, para la crianza, resulta acertado recoger el paradigma asumido en la actualidad en el ámbito de la calidad de vida familiar y la discapacidad intelectual, perfectamente extrapolable al ámbito general de la atención temprana, el cual apuesta por una reconsideración del rol de las familias. El énfasis ya no está ni en pretender "arreglar" a la persona con discapacidad, ni exclusivamente en la madre, ni tampoco en la calidad de los servicios. El énfasis se sitúa en la calidad de vida de cada uno de los componentes y de la familia como un todo (Verdugo, Rodríguez y Sainz, 2012).

Es así como se plantea en el Modelo de Capacitación ('empoderamiento') Familiar o Modelo de Calidad de vida
Familiar de Turnbull (2003) que representa los nuevos retos en los que el punto de mira se dirige a lograr la participación familiar activa mediante la capacitación familiar, es decir, promoviendo que sea la propia familia la que sepa reconocer sus prioridades y elaborar su propio plan para alcanzarlas.

\section{Modelo de intervención con la familia}

Antes de definir el modelo de intervención con la familia, volvemos a subrayar la idea de que los progenitores son participantes activos en el proceso educativo y rehabilitador de sus hijos/as, por lo que es necesario incorporarles al ámbito de actuación (Giné et al., 2009), es decir, que ellos constituyen una pieza clave en la propia intervención. Según Dunst (2004) es muy importante que las familias participen, por ello, hay que ayudarles a modificar, adquirir, cambiar, mejorar y ajustar algunas pautas o estrategias de interacción que ejercen en sus casas diariamente con los menores para que el trabajo de atención temprana pueda considerarse completo. El objetivo pasa de priorizar la atención a los padres, a aprender a trabajar con los padres (Giné et al., 2009).

El modelo de intervención con la familia más adecuado es el modelo de entornos competentes, este modelo constituye un modelo ecológico de intervención basado en la necesidad de conseguir que los padres potencien la percepción de auto-competencia (Perpiñán, 2003, 2009), que las familias cada vez sean más competentes enriqueciendo adecuadamente el ambiente en el que se desarrollan los niños/as, adecuando estímulos, condiciones ambientales, evitando riesgos y desarrollando un adecuado clima emocional:

Un enfoque ecológico nos ayuda a comprender que el desarrollo del niño/a resulta de la interacción de lo biológico y lo social; es decir, de su potencial orgánico en desarrollo, de la forma en que actúa en el mundo y de cómo éste le trata (De Linares y Rodríguez, 2004, p. 343).

$\mathrm{Al}$ analizar las variables que influyen en el buen desarrollo familiar, se aprecia que dichas variables son las que propone Perpiñán (2003): estructura, historia, estilo educativo, sistema de atribuciones, emociones, expectativas y necesidades.

La familia es todo un entramado de emociones, necesidades, expectativas y estilo educativo que influyen decisivamente en su adecuado funcionamiento. Muchos de estos aspectos se ven beneficiados al trabajar los profesionales de la atención temprana con las familias, y ayudarles a adquirir esas competencias necesarias para hacer frente a las situaciones en las que se encuentran día a día con sus hijos/as. Pero debido a la experiencia, se puede apreciar que no es suficiente, las familias necesitan un entramado social que de cobertura a la nueva situación que están viviendo.

Las familias son un objeto directo de intervención, tienen unas necesidades concretas de apoyo, información, formación y asesoramiento (Díez, 2008) que deben ser cubiertas por los profesionales, pero también pueden ser cubiertas con la ayuda de otras familias en su situación. Porque, el objetivo final es capacitar y habilitar a las familias para que puedan funcionar 
de manera efectiva en sus contextos sociales (Leal, 1999), siendo importante, ayudarles a solidificar una red social con otras familias con intereses comunes. Para ello es fundamental conocer sus demandas, sus estilos de enfrentamiento a las situaciones, ayudarles a responder de manera adecuada, y sobre todo que ellos sepan hacer uso de los apoyos sociales de los que pueden contar.

En definitiva, se puede considerar que existen dos ideas básicas subyacentes en cualquier intervención: hay que analizar y tratar a las familias como un todo y son fundamentales los contextos y el uso de redes de apoyo sociales (De Linares y Rodríguez, 2004).

\section{Apoyo familiar: establecimiento de redes sociales}

Como ya se ha indicado anteriormente un trabajo centrado en la familia, implica que se les proporcione una red social de referencia y apoyo, para que tanto los niños/as con necesidades como sus familias se sientan integrados en la comunidad (Turnbull et al., 2007). Tener buenas relaciones sociales va a mejorar la calidad de vida de estas familias, entendiendo la calidad de vida desde una concepción integral (Córdoba, Gómez y Verdugo, 2008). En esta concepción se visualiza a la familia como una unidad de apoyo, trabajando en equipo con los profesionales de atención temprana y con otras familias, en la búsqueda de logros que respondan a sus necesidades y sus expectativas (Schalock y Verdugo, 2002).

Los programas puestos en marcha para intervenir en el núcleo familiar, partiendo de Fernández (2011), se pueden sintetizar en: individuales y de participación en las sesiones de intervención. En los programas individuales se encuentran los de acogida y de seguimiento individual. El programa de acogida se centra en un conjunto de acciones que se dan en el primer momento del proceso y que son muy importantes para poder establecer el marco de intervención. Dentro de este programa podemos encontrar dos subprogramas que son "Primera noticia" y "Padre a padre".
En el ámbito de la atención temprana se conoce como "primera noticia" el momento en que un profesional da cuenta a los padres de que su hijo/a presenta una anomalía congénita, una discapacidad, un trastorno o un retraso del desarrollo. Una información que los padres reciben casi siempre con mucho dolor y que además, puede tener efectos de alcance sobre el proyecto familiar y sobre el futuro de los menores (Federación Estatal de Asociaciones de Profesionales de Atención Temprana-GAT, 2011). Este programa intenta proporcionar información para resolver una situación de incertidumbre, producir cambios constructivos en la situación vital de las familias tan rápido como sea posible y cimentar un guión de esperanza. Normalmente son médicos los que se encargan de dar esta primera noticia, pero en ocasiones son los mismos profesionales de atención temprana quienes se encargan de darla.

Y el programa "Padre a padre", posiblemente el más interesante desde el punto de vista de nuestro objetivo. Consiste en una serie de visitas que llevan a cabo otras familias que han pasado por una situación similar, así se pueden identificar con ellas, consultar dudas y apreciar cómo han podido superar la nueva situación: "Muchos padres manifiestan cómo los primeros contactos con otros padres que han experimentado y experimentan una situación parecida, con un hijo/a con el mismo tipo de discapacidad, fueron muy significativos y les aportaron información y ayuda desde una situación natural" (Perpiñán, 2009, p. 234). Por otra parte, los programas de seguimiento individual engloban los "seguimientos familiares" y "contactos puntuales". Por último, está el programa de participación en las sesiones de intervención. Este programa consiste en que los padres estén presentes en algunas sesiones que tienen sus hijos/as con los profesionales de la atención temprana, para así asegurar una verdadera comprensión de las capacidades de sus hijos/as.

Se podría realizar una reflexión sobre si estos programas cubren o no lo que han identificado como factores de protección en el tránsito a la vida familiar. Estos factores, y también los de riesgo, se recogen en la Tabla 1.

Tabla 1

Factores de protección y de riesgo en el tránsito a la vida familiar (Ponte et. al, 2008).

\begin{tabular}{|c|c|}
\hline Factores de Protección & Factores de Riesgo \\
\hline $\begin{array}{l}\text { Conocer y escuchar las preocupaciones } \\
\text { de la familia, distinguir los temas que nos plantean abiertos a nuestra opinión y los } \\
\text { que consideran cerrados }\end{array}$ & $\begin{array}{l}\text { Anticipar los temas sin considerar la posición de la familia en función de ideas } \\
\text { preestablecidas }\end{array}$ \\
\hline $\begin{array}{l}\text { Descentralizar, sectorizar los servicios para acercarlos a la familia y su entorno, } \\
\text { incluyendo el apoyo domiciliario }\end{array}$ & $\begin{array}{l}\text { Concentración institucional de los servicios, asilando a las familias de su } \\
\text { entorno, generando costes y viajes innecesarios }\end{array}$ \\
\hline Realizar las citas externas imprescindibles & Reiterar pruebas, historias, revisiones \\
\hline Destacar los aspectos coherentes y evitar descalificaciones & Destacar la disparidad o descalificar a la familia o a otros profesionales \\
\hline Ofrecer flexibilidad, estabilidad y disponibilidad para las demandas & Listas de espera, demora en las citas \\
\hline Temporizar dudas y cuidar los pronósticos & Prolongar situaciones de duda o anticipar resultados dudosos o inciertos \\
\hline Aclarar los significados de los informes o comentarios cuando resulten opacos & Dar los significados por sabidos \\
\hline $\begin{array}{l}\text { Abrir espacios para la reflexión y el diálogo interno de los padres sobre temas de la } \\
\text { vida cotidiana }\end{array}$ & $\begin{array}{l}\text { Entrometerse en asuntos en que la familia no solicita mediación, aun con el } \\
\text { consentimiento de uno de los padres }\end{array}$ \\
\hline Ofrecer espacios de apoyo emocional: grupos de padres & $\begin{array}{l}\text { Establecer alianzas o informar de manera privilegiada a una de las partes de la } \\
\text { familia }\end{array}$ \\
\hline $\begin{array}{l}\text { Diálogo activo y negociación de conflictos } \\
\text { Apoyo económico, laboral, tiempo, redes } \\
\text { Acompañar el transito a la escuela }\end{array}$ & $\begin{array}{l}\text { Descalificación encubierta de uno de los padres (falta de atención, gestos, } \\
\text { comentarios) }\end{array}$ \\
\hline
\end{tabular}


Nuestro problema de investigación surge a propuesta de un Centro de Atención e Intervención Temprana (CAIT) generalista, que atiende a niños/as con trastornos en el desarrollo o riesgo de padecerlos, independientemente del tipo de trastornos que sea. La Tabla 2 aporta información sobre el número de niños y niñas que están recibiendo tratamiento en la actualidad, así como el perfil y necesidades.

Tabla 2

Perfil de usuarios del Centro de Atención e Intervención Temprana.

\begin{tabular}{lc}
\hline \multicolumn{1}{c}{$\begin{array}{c}\text { Perfil de usuarios del Centro de Atención e } \\
\text { Intervención Temprana }\end{array}$} & $\begin{array}{c}\% \text { Población } \\
\text { atendida }\end{array}$ \\
\hline Niños/as con Trastornos del Espectro Autista predominantes & $13.90 \%$ \\
\hline Niños/as con Trastornos sensoriales predominantes & $1.30 \%$ \\
\hline $\begin{array}{l}\text { Niños/as con Trastornos del Desarrollo con Discapacidad } \\
\text { Motora predominantes }\end{array}$ & $14.50 \%$ \\
\hline $\begin{array}{l}\text { Niños/as con Trastornos del Desarrollo con Discapacidad } \\
\text { Intelectual predominante }\end{array}$ & $13.30 \%$ \\
\hline Alto Riesgo: Seguimiento del Recién Nacido de Alto Riesgo & $23.20 \%$ \\
\hline $\begin{array}{l}\text { Trastorno del habla y del lenguaje: Niños/as con Trastornos } \\
\text { del Habla y/o del Lenguaje predominante }\end{array}$ & $33.80 \%$ \\
\hline
\end{tabular}

Este centro está insertado en una asociación, que atiende a 152 niños/as y sus familias. Cuenta con los espacios adecuados tanto para la intervención de los menores, como para llevar a cabo las acciones dirigidas a la familia, para ello toma como principio fundamental la definición que recoge el Libro Blanco de Atención Temprana (2000):

Atención Temprana es el conjunto de intervenciones, dirigidas a la población infantil de 0 a 6 años, a la familia y al entorno, que tienen por objetivo dar respuesta lo más pronto posible a las necesidades transitorias o permanentes que presentan los niños con trastornos en su desarrollo o que tienen el riesgo de padecerlos. Estas intervenciones, que deben considerar la globalidad del niño, han de ser planificadas por un equipo de profesionales de orientación inter y transdisciplinar (p. 12).

Esta asociación se constituyó en Abril de 1977, por un grupo de padres y madres preocupados por sus hijos/as, con el deseo de brindarles rehabilitación, el derecho a la educación y atención integral. Entre los diferentes servicios de apoyo a usuarios y familia ocupa un lugar importante el servicio de atención temprana, como unidad orgánica y funcional dotada de una infraestructura y material con ubicación identificable.

Las familias que acuden al CAIT son derivadas por su pediatra de referencia o por el Equipo Provincial de Atención Temprana. Una vez realizada la derivación, la familia se pone en contacto con la trabajadora social, para concretar una cita y posterior valoración, ya sea para pasar a lista de espera o para iniciar la intervención. En el primer caso, una vez realizada la valoración, se elabora un documento que recoge los aspectos más significativos de la entrevista a la familia y evaluación realizada al niño/a, existiendo un protocolo de coordinación con el Departamento de Atención Tempana de la Consejería de Igualdad, Salud y Política Social, de la Delegación de nuestra provincia. En el segundo, se inicia la intervención con una entrevista inicial a la familia, donde se pretende conocer el estado emocional, situación laboral y social, tipo de familia y nivel estudios. También se le informa del compromiso de los profesionales de coordinarse con el resto de los contextos en los que se desarrolla el niño/a.

Las familias que acuden al CAIT en su mayoría son de clase media (69\%), esta categoría la forman familias con trabajos estables de los dos o uno de los progenitores. Respecto a nivel de estudios predomina las personas que han finalizado los elementales y con especializaciones específicas, estudios medios o diplomaturas. A continuación, son las denominadas familias con riesgo de exclusión social las más numerosas $(20 \%)$. Éstas pueden ser aquellas que pertenecen a grupos con dificultades de acceso a recursos sociales, o que en la mayoría de los casos no han finalizado los estudios primarios, o bien que están un periodo transitorio de especiales dificultades económicas como puede ser, enfermedad, paro de los dos progenitores, etc. Le siguen, en menor número (7.90\%), las familias de clase media-alta, poseen estudios superiores y que suelen ejercer la profesión para la que se han preparado. Por último, están las "otras familias" por su situación específica, constituyendo el $3.10 \%$ de la población atendida.

La intervención familiar se realiza de forma individualizada, haciéndoles partícipes de la misma, facilitando información sobre recursos administrativos, asistenciales, económicos y legales, así como facilitar el contacto con otras asociaciones. Además a lo largo del año se realizaran talleres grupales. Nuestra experiencia evidencia que los talleres grupales no funcionan, ya que hay escasa participación y suelen ser siempre las mismas familias. El programa de evaluación familiar es paralelo al del menor, y ante signos de alarma, se informa de la importancia de contactar con un especialista, facilitando el contacto con dichos especialistas. Todas estas actuaciones están coordinadas y protocolarizadas desde el Departamento de Atención Tempana de la Consejería de Igualdad, Salud y Política Social, de la Delegación provincial.

El objetivo de este estudio es conocer las necesidades reales de las familias que acuden a este CAIT generalista con la finalidad de diseñar un programa que responda a las mismas. Este objetivo ha derivado en las siguientes hipótesis:

$\mathrm{Ho}=$ las familias con más de un familiar con discapacidad tienen el mismo interés hacia participar en un programa de familias, que aquellas familias que no tienen otros familiares con discapacidad; $\mathrm{Ho}=\mu 1=\mu 2$

$\mathrm{H} 1=$ las familias con más de un familiar con discapacidad no tienen el mismo interés en participar en un programa de familias que las familias que no tienen más familiares con discapacidad; $\mathrm{H} 1=\mu 1 \neq \mu 2$.

\section{Participantes}

\section{Método}

Los participantes han sido 58 familias atendida por el CAIT en diciembre de 2014. Su selección ha sido mediante un muestreo aleatorio. La población total es de 152 familias. 


\section{Instrumentos}

Para identificar las necesidades de las familias atendidas en el CAIT se ha elaborado un instrumento de recogida de información, concretamente un cuestionario de satisfacción. El cuestionario se estructura en tres partes, una primer parte de preguntas identificativas, una segunda parte, o cuerpo del instrumento, desde el ítem 1 al ítem 18, centrados en una escala de tipo Likert con 5 opciones de respuesta que van desde nunca a siempre. Y una tercera parte, o pie del cuestionario, de preguntas abiertas desde la pregunta 19 a la 22 , donde las familias tienen absoluta libertad en sus respuestas.

La elaboración de los ítems se ha fundamentado en las dimensiones e indicadores de calidad de vida familiar según el Beach Center (citado por Verdugo, Rodríguez y Sains, 2012), las variables a tener en cuenta en el sistema familiar de Perpiñan (2003), los factores de protección y de riesgo en el tránsito a la vida familiar (Ponte et al., 2008), así como en la propia experiencia de los trabajadores del CAIT.

\section{Procedimiento}

Para dar respuesta al objetivo planteado en este trabajo, se ha puesto en práctica un estudio no experimental, descriptivo y ex post facto, donde lo que se ha pretendido es conocer las necesidades de las familias, de tal forma que se pueda diseñar e implementar un programa de intervención familiar de acuerdo a las necesidades de las mismas.

Para el análisis de los datos se ha utilizado el paquete estadístico SPSS V.20. En primer lugar se ha realizado un análisis descriptivo de los datos, para posteriormente realizar un análisis inferencial. Para el contraste de hipótesis se ha realizado una comparación de medias, teniendo en cuenta las hipótesis enunciadas en la introducción.

Dicho contraste se realizó mediante la prueba $t$ de Student para dos muestras independientes.

Por último se realizó un análisis cualitativo de las preguntas abiertas establecidas en el pie del cuestionario.

\section{Resultados}

De las 58 familias encuestadas el $74.14 \%$ eran mujeres y el $24.14 \%$ hombres, un $1.70 \%$ no respondió a este ítem. Respecto a la edad es variable, oscila entre los 25 y los 67 años, siendo la media de 39.25 años.

En la mayoría de los casos quien responde al cuestionario es la madre, en un $65.50 \%$ de los casos, el padre en un $22.40 \%$, la abuela en un $6.90 \%$ y el abuelo en un $3.40 \%$. Las familias poseen un nivel de estudios medios o superiores, concretamente un $75.86 \%$. El $82.80 \%$ son familias tradicionales, con una media de 3.73 miembros por familia. Principalmente la noticia de la discapacidad se la da a las familias el pediatra de atención primaria (en un $36.20 \%$ de los casos), seguido de los profesionales del propio hospital donde se produce el nacimiento $(24.10 \%)$ o incluso son los miembros de los centros de atención temprana los que dan dicha noticia a las fami- lias (17.20\%). Por otro lado, un $29.30 \%$ de estas familias sí poseen otro familiar directo con algún tipo de discapacidad, frente a un $62.10 \%$ que afirman no poseer familiares con dichas peculiaridades.

Según estas familias cuando recibieron la noticia de la discapacidad del menor afloraron muchos sentimientos frente a dicha situación, de todos los sentimientos posibles el más enfatizado por los familiares fue la preocupación (39.70\%), aunque hay que tener en cuenta que muchos de ellos también identificaron los de: confusión, frustración miedo, culpa y depresión. También es de resaltar que para el $8.60 \%$ el sentimiento predominante fue la felicidad por la llegada de un nuevo miembro. $\mathrm{Y}$ que ninguna de estas familias marcaron sentimientos como indiferencia u ocultamiento.

Las familias encuestadas afirman que "a veces" se sienten comprendidos por otras familias que no tienen hijos/as con necesidades educativas especiales, concretamente el $32.80 \%$. Por otro lado, observando la Figura 1 se aprecia que sí se siente "casi siempre" y "siempre" comprendidos por otras familias que sí tienen hijos/as con algún tipo de discapacidad.

\section{Figura 1}

Grado en que las familia encuestadas se sienten comprendidas por otras familias con hijos/as con necesidades educativas especiales.

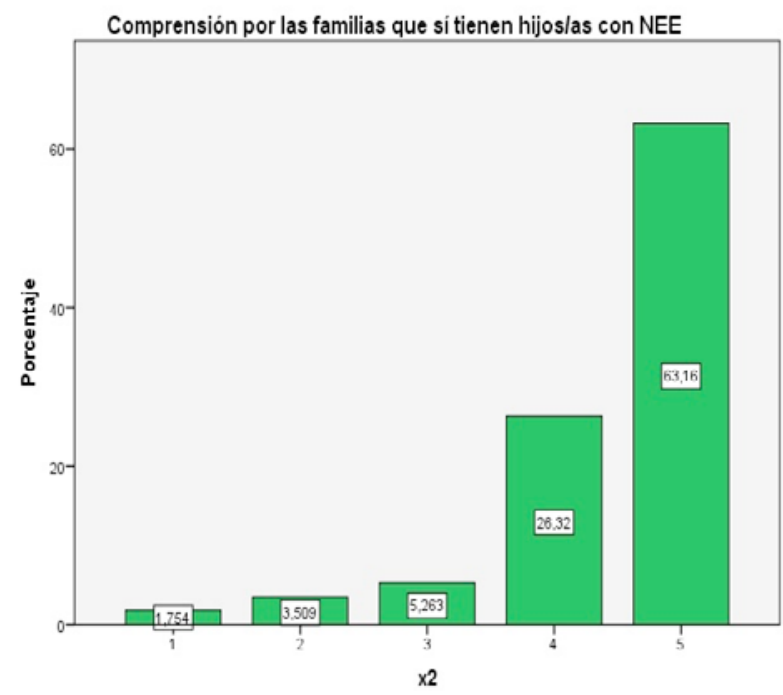

Nota. 1: Nunca; 2: Casi nunca; 3: A veces; 4: Casi siempre; 5: Siempre.

Aunque un $48.80 \%$ afirma no tener ningún contacto con otras familias del centro de atención temprana, y un $17.24 \%$ indican que "casi nunca" tienen ese tipo de contacto. Para un $36.21 \%$ de estas familias "a veces" les gustaría mantener contacto con dichas familias, y a un $29.31 \%$ "siempre".

Al plantearles si poseen el apoyo necesario para aliviar el estrés existen disparidad de opiniones, como se muestra a continuación en la Figura 2. No obstante, el valor más sobresaliente es el 5 , lo cual indica que un $27.59 \%$ siente que "siempre" cuentan con dicho apoyo. 
Figura 2

Grado en que las familias encuestadas consideran que cuentan con el apoyo social necesario para aliviar el estrés.

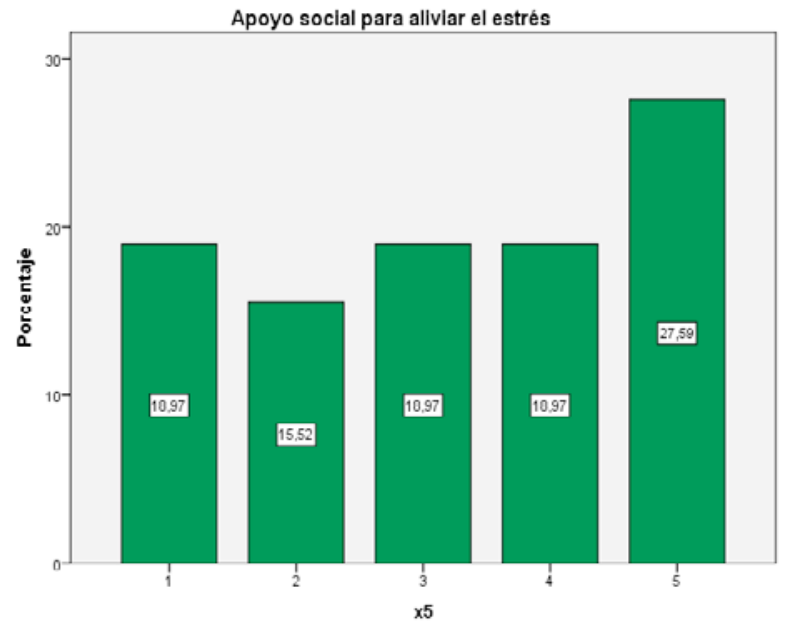

Nota. 1: Nunca; 2: Casi nunca; 3: A veces; 4: Casi siempre; 5: Siempre.

El 48.18\% de estas familias afirman que "nunca" asisten a las charlas o talleres que organiza el CAIT de apoyo a las familias, frente a un $18.52 \%$ que afirman que "siempre" lo hacen. Y un $25.86 \%$ afirman que les gustaría participar en la organización de dichos talleres. El contenido de dichas charlas, según estas familias "siempre" es de mucho interés (57.69\%).

Cuando les dieron las noticias a estas familias a un elevado porcentaje de las mismas les hubiera gustado contar con el apoyo de otras familias que hubieran pasado por la misma situación, como se muestra en la Figura 3.

\section{Figura 3}

Grado en que a las familias encuestadas les hubiera gustado contar con el apoyo de otras familias que hubieran pasado por la misma situación cuando les dieron la noticia de que su hijo/a hija presentaba una discapacidad.

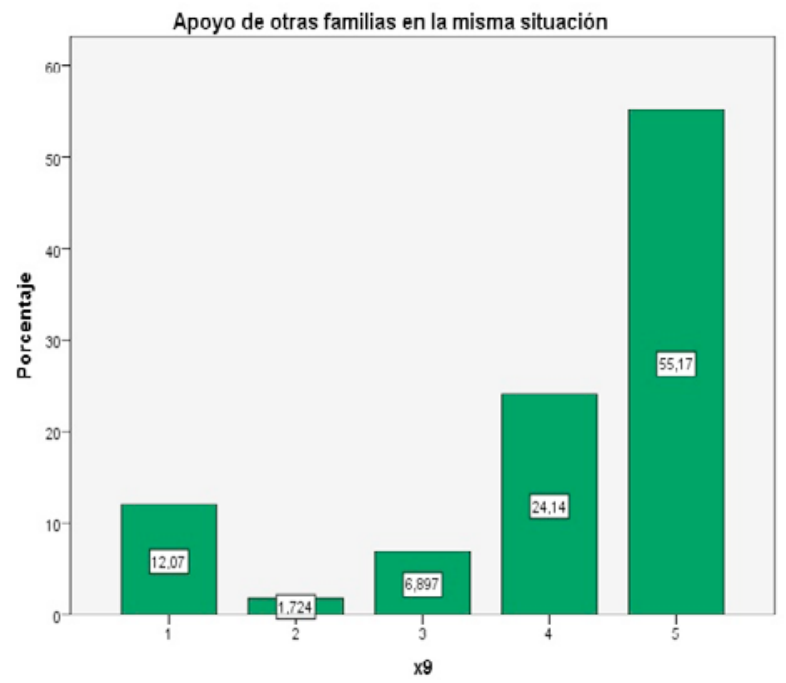

Nota. 1: Nunca; 2: Casi nunca; 3: A veces; 4: Casi siempre; 5: Siempre.
Estas familias en un $74.14 \%$ piensan que sería necesario que existiera una asociación de familias que orientara y apoyara a las nuevas familias que se encuentran con un hijo/a con necesidades educativas especiales. Y un $55.70 \%$ afirman que la propia entidad de atención temprana tendría que facilitar espacios para ese intercambio, es más, para un $67.24 \%$ dichos espacios tendrían que ser permanentes.

El $68.97 \%$ de las familias encuestadas piensan que familias bien formadas pueden ser un buen estímulo para otras familias respecto a sus estilos educativos, y les pueden servir para entender lo que les ha tocado vivir $(66.67 \%)$. Estar en contacto con otros padres ante la misma situación les permite a estas familias siempre expresar emociones, desahogos y reencontrar la dirección (60.34\%). Al plantear a estas familias si los profesionales se centran más en los niños/as que en las familias existen discrepancias de opiniones, como se puede apreciar en la Figura 4. El valor predominante es el 3, es decir, "a veces" el interés de los profesionales del CAIT se centra en los menores. Y al 35.09\% les gustaría que se dispusiera de más tiempo para atender a las familias.

\section{Figura 4}

Grado en que las familiar encuestadas consideran que los profesionales se centran más en los niños/as que en las familias.

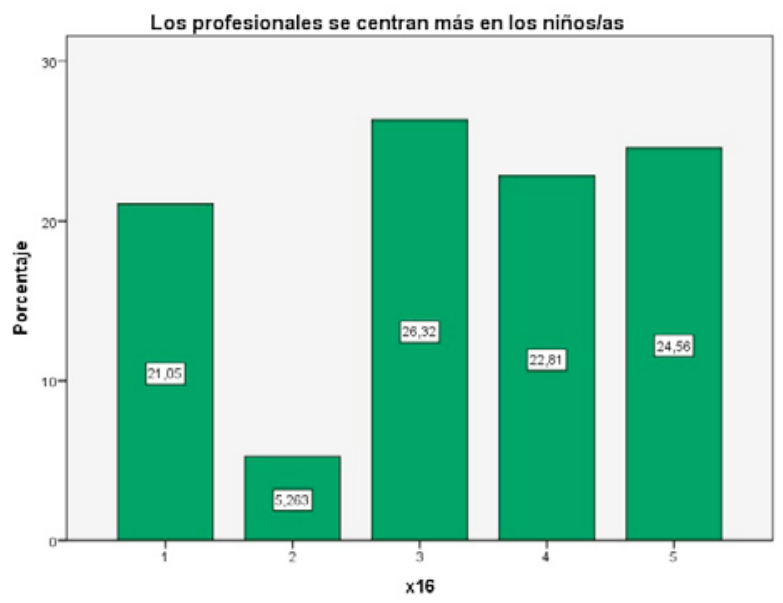

Nota. 1: Nunca; 2: Casi nunca; 3: A veces; 4: Casi siempre; 5: Siempre.

Dentro de este bloque se planteó a las familias sí ellos se consideraban capacitados para recibir la formación necesaria para ser familia acogedora, y las respuestas fueron dispares (Figura 5). Casi están igualadas las familias que piensan que no están nada capacitadas, un $32.76 \%$, frente a otras familias que piensan que sí están capacitadas para recibir dicha formación, concretamente el $29.31 \%$.

Al realizar el contraste de hipótesis se acepta la hipótesis nula, puesto que no hay diferencias significativas en el contraste de medias, lo cual indica que tanto las familias que tienen hijos y otros familiares con discapacidad, como aquellas familias que sólo tienen el miembro que acude al CAIT con discapacidad tienen el mismo interés en que exista un programa de familias (Tabla 3). 
Figura 5

Grado en que las familias encuestadas manifiestan sentirse capacitadas para recibir la formación necesaria para ser familia acogedora.

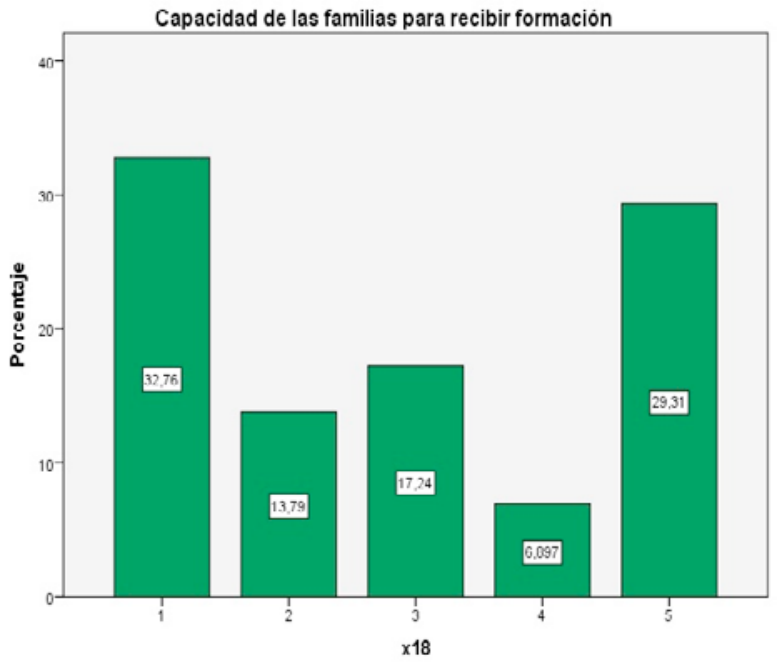

Nota. 1: Nunca; 2: Casi nunca; 3: A veces; 4: Casi siempre; 5: Siempre.

Tabla 3

Contraste de medias entre familias con más de un miembros con discapacidad y familias con un único miembro con discapacidad.

\begin{tabular}{llcccccc}
\hline & Otros_miembros & $N$ & $M$ & $D T$ & g.l. & $t$ & $p$ \\
\hline \multirow{2}{*}{ Ítem 10 } & Con discapacidad & 17 & 4.53 & .943 & 51 &,- 336 &, 738 \\
& No discapacidad & 36 & 4.61 & .766 & & & \\
& Si discapacidad & 17 & 2.88 & 1.833 & 51 & \multirow{2}{*}{044} & \multirow{2}{*}{.965} \\
& No discapacidad & 36 & 2.86 & 1.552 & & & \\
\hline
\end{tabular}

Nota. Item 10: Necesidad de una asociación de padres que orientara y apoyara a las nuevas familias que se encuentran con un hijo/a con necesidades educativas especiales. Item 18: Capacidad de las familias para recibir la formación necesaria para ser familia acogedora.

Por último, se plantearon tres preguntas abiertas en las cuales ellos tenían la opción de contestar libremente. Las respuestas fueron categorizadas, obteniéndose los siguientes resultados:

a) Sobre cuáles pensaban ellos que deberían ser las cualidades o características que deberían de tener unos padres para ser "familias acogedoras", utilizando la propuesta del programa "Padre a Padre", pensaban que deberían ser: "Empáticos" (para 20 de las familias encuestadas), "tener mucha paciencia" (para 13 familias), "estar bien formados" y "ser cariñosos" (para 9 familias), "comprensivos" (para 8 familias) y "tener disponibilidad" (para 7 familias). Además de otras cualidades resaltadas por algunas familias como: "ser flexibles", "tener asumida su situación", "mantener una visión positiva", "realistas", "ser agradables", "tolerantes", "integradores" y "cercanos".

b) Para las familias encuestadas dichas familias acogedoras les pueden aportar sobre todo "experiencia", "ароуо emocional", "seguridad" y "tranquilidad". c) Y la motivación para participar como familias acogedoras o acompañantes puede ser: "ayudar a los demás", "amor hacia los niños/as" y "tener tiempo".

\section{Discusión}

En nuestro caso parece que no se está dando respuesta a las acciones necesarias en relación con las familias, sobre todo si tenemos en cuenta las recomendadas por la Federación Estatal de Asociaciones de Profesionales de Atención Temprana-GAT (2005) y que se sintetizan en:

a) Creación de marcos de participación familiar en la toma de decisiones y elección de un Coordinador Asesor de la familia.

b) Apoyo emocional, social, informativo y formativo relacionado con la información diagnóstica o trastorno del desarrollo infantil de su hijo.

c) Información, orientación y apoyo relacionado con el desarrollo personal del niño y sus necesidades cotidianas.

d) Apoyo a la reorganización del conjunto de la unidad de convivencia.

e) Orientación sobre otros apoyos económicos, sanitarios, educativos y sociales.

f) Información sobre asociaciones de padres, usuarios y otras relacionadas.

Una interpretación general de los datos obtenidos debe hacernos buscar una respuesta a una situación incoherente, ya que las familias entienden que tener relación otras en la misma situación, le ofrecería apoyo emocional, seguridad y tranquilidad. Sin embargo, casi la mitad de ellas reconocen no tener contacto y un $48.18 \%$ no participan en los talleres para familias organizados por el centro.

A partir de esta idea principal, los resultados comprueban algunos de los ya alcanzados en trabajos anteriores. Por ejemplo, los referidos a los sentimientos de las familias. Es el de preocupación es el más repetido por las familias encuestadas, además de los de confusión, frustración, miedo, culpa y depresión. Los resultados ponen de manifiesto las ideas recogidas en el trabajo de Díez (2008) y de Perpiñán (2003). O también el cómo para paliar estos sentimientos, indicando que se sienten comprendidos por otras familias con hijos/as con algún tipo de discapacidad. Esto confirma la idea de la necesidad de apoyo, información, formación y asesoramiento de Díez (2008) y la aportación de información y ayuda de Perpinán (2009) planteadas de manera más amplia en la introducción de nuestro trabajo. Y por último, las familias entienden que deben ser los CAIT los que faciliten espacios para los encuentros familiafamilia, siguiendo las indicaciones de propuestas como las de Schalock y Verdugo (2002) o el libro Blanco de Atención Temprana (2000) recogidas anteriormente. Además el 67.24\% añade que debería ser un espacio permanente.

Valorando e interpretando estos resultados, entendemos que poner en marcha un programa de apoyo para y entre familias puede ser más fácil desde un centro de atención infantil 
temprana, ya que en este momento están totalmente volcadas en sus hijos/as, y es a través de la atención a sus hijos/as como se puede facilitar a las familias los apoyos necesarios, brindándoles espacio emocional y de comunicación entre familias con más experiencia en atención a personas con necesidades especiales, y nuevas familias que están iniciando este duro recorrido, que en función de los recursos personales y apoyos que encuentren en su entorno, puede ser más o menos largo.

La tarea ahora es diseñar e implementar el programa que ofrezca una respuesta acertada a la demanda de las familias, siendo el denominado "Padre a Padre" el más adecuado para utilizarlo como referente y teniendo también en cuenta las características definidas por las familias de nuestro estudio.

\section{Referencias}

1. Aranda, R. y De Andrés, C. (2004). La organización de la Atención Temprana en la educación infantil. Tendencias Pedagógicas, 9, 217-246. Recuperado el 22-10-201, de http://www.tendenciaspedagogicas.com/ Articulos/2004_09_10.pdf

2. Córdoba, L.; Gómez, J. y Verdugo, M.A. (2008). Calidad de vida familiar en personas con discapacidad: un análisis comparativo. Universitas Psychologica, 7, 369-382. Recuperado el 17-11-2015, de http://revistas.javeriana.edu.co/ index.php/revPsycho/article/view/369

3. Cunningham, C. (1995). El sindrome de Down. Una introducción para padres. Barcelona: Paidós.

4. De Linares Von Schmiterlöw, C. y Rodríguez, T. (2004). Bases de la intervención familiar en Atención Temprana. En J. Pérez-López y A. Brito (Coord.), Manual de Atención Temprana (pp. 333-351). Madrid: Pirámide.

5. Díez Martínez, A. (2008). Evolución del proceso de Atención Temprana a partir de la triada profesional-familianiño. Revista Síndrome de Down, 25, 46-55. Recuperado el 6-10-2015, de http://www.downcantabria.com/revistapdf/97/46-55.pdf

6. Dotor, M. y Fernández, E. (2009). Guía de diseño y mejora continua de procesos asistenciales. Proceso Asistencial Integrado. Sevilla: Consejería de Salud Junta de Andalucía. Recuperado el 11-11-2015, de http://www. juntadeandalucia.es/salud/sites/csalud/contenidos/Informacion General $/ \mathrm{p} 3$ p 3 procesos asistenciales integrados/ guia diseno mejora procesos?perfil =org

7. Dunst, C.J. (2004). Revisiting Rethinking Early Intervention. En M.A. Feldman (Ed.), Early Intervention. The essentials readings (pp. 262-283). EE.UU: Blackwell Publishing. $\quad$ http://dx.doi.org/10.1002/9780470755778. $\underline{\operatorname{ch} 10}$

8. Federación Estatal de Asociaciones de Profesionales de Atención Temprana-GAT (2005). Libro Blanco de la Atención Temprana. Madrid: Real Patronato sobre Discapacidad. Recuperado el 12-12-2015, de https://www.fcsd.org/ fichero-69992_69992.pdf
9. Federación Estatal de Asociaciones de Profesionales de Atención Temprana-GAT (2005). Recomendaciones técnicas para el desarrollo de la Atención Temprana. Madrid: Real Patronato sobre Discapacidad. Recuperado el 12-122015, de http://www.atenciontemprana.com/EIDocumentsAT/Recomendaciones $\% 20 \mathrm{~T} \%$ E9cnicas.pdf

10. Federación Estatal de Asociaciones de Profesionales de Atención Temprana-GAT (2011). La primera noticia. Estudio sobre los procedimientos profesionales, las vivencias $y$ las necesidades de los padres cuando se les informa de que su hijo tiene una discapacidad o un trastorno del desarrollo. Madrid: Real Patronato sobre Discapacidad.

11. Fernández, M. M. (2011). Atención Temprana. Trabajo Fin de Máster. Almería: Universidad de Almería. Recuperado el 5-12-2015, de http://repositorio.ual.es/jspui/ bitstream/10835/1160/1/Fernandez_\%20Garcia_Maria del \%20Mar.pdf

12. Giné, C., Gràcia, M., Vilaseca, R. y Balcells, A. (2008). Trabajar con las familias en Atención Temprana. Revista interuniversitaria de formación del profesorado, 65, (23,2), 95-113. Recuperado el 4-12-2015, de http://www.aufop. com/aufop/uploaded_files/revistas/124856066610.pdf

13. Leal, L. (1999). A family-centered approach to people with mental retardation. Washington: American Association on Mental Retardation.

14. Perpiñán, S. (2003). La intervención con familias en los programas de AT. En I. Candel (Dir.), Atención temprana. Niños con síndrome de Down y otros problemas de desarrollo (pp. 57-79). Madrid: Feisd.

15. Perpiñán, S. (2009). Atención temprana y familia. Cómo intervenir creando entornos competentes. Madrid: Narcea.

16. Ponte, J. et. al. (2008). Guía de estándares de calidad en Atención Temprana (Revisión 2004). Madrid: Real Patronato sobre Discapacidad.

17. Schalock, R. y Verdugo, M. (2002). The concept of quality of life in human services. A handbook for human service practitioners. Washington: American Association on Mental Retardation.

18. Silvestre, N. (2010). Las familias con criaturas con necesidades educativas especiales: las familias que oyen pero tienen criaturas sordas. Educar, 45, 99-115. Recuperado el 12-11-2015, de http://educar.uab.cat/article/view/96

19. Trenado, R.; Pons-Salvador, G. y Cerezo, M.A. (2009). Proteger a la infancia: apoyando y asistiendo a las familias. Papeles del Psicólogo, 30, 24-32. Recuperado el 6-122015, de http://www.papelesdelpsicologo.es/vernumero. asp?id $=1653$

20. Turnbull, A. (2003). La calidad de vida de la familia como resultado de los servicios: el nuevo paradigma. Siglo Cero, 207, 59-73. Recuperado el 5-12-2015, de http://www. feaps.org/archivo/publicaciones-feaps/siglo-cero/numerosgratuitos/364-n-207.html

21. Turnbull, A.P., Turnbull, H.R., Erwin, E. y Soodak, L. 
(2007). Families, professionals and exceptionality. Positive outcomes though partnership and trust ( $\left.5^{\mathrm{a}} \mathrm{Ed}\right)$. Upper Saddle River, NJ: Merrill/Prentice Hall.

22. Verdugo, M.A., Rodríguez, A. y Sainz, F. (2012). Escala de calidad de vida familiar. Manual de aplicación. Salamanca: INICO. Recuperado el 4-12-2015, de http://sid.usal.es/ libros/discapacidad/26164/8-1/escala-de-calidad-de-vidafamiliar-manual-de-aplicacion.aspx

Fecha de recepción: 11 de enero, 2015

Fecha de recepción de la versión modificada: 15 de abril, 2015

Fecha de aceptación: 11 de junio, 2015 